\title{
DUALITIES IN FRACTIONAL STATISTICS
}

\author{
M. I. BECIU \\ Department of Theoretical Physics, Institute of Atomic Physics \\ Magurele, Sect 6, P.O. Box MG-6, Bucharest, Romania \\ Department of Physics, Technical University B-d Lacul Tei 124, Bucharest, Romania \\ beciu@mail.utcb.ro
}

November 17, 2018

\begin{abstract}
We first reobtain in a simpler way the Haldane fractional statistics at thermal equilibrium using an interpolation argument. We then show that the mean occupation number for fractional statistics is invariant to a group of duality transformations, a nonabelian subgroup of fractional linear group.
\end{abstract}

\section{Introduction}

In the two-dimensional systems the particles are not constrained to obey the Fermi or Bose statistics but they may have fractional statistics; two well known exemples are offered by the quasi-particles in the fractional quantum Hall effect and by anyons. Prompted by this, Haldane 1] stated a generalized exclusion principle that allows for intermediate statistics, though his derivation of the particle occupation number does not involve the spatial dimensionality and so, it seems to be valid for an arbitrary number of dimensions. Wu 2 discussed the thermodynamics of the fractional statistics particles (geons) in the ideal gas approximation. Further developments on the thermodynamical aspects of geons can be found in [3], [4. In an akin paper [5] to the present one, it was shown that the distribution funtion for geons enjoys a duality property. Quite recently, a number of authors [6], starting from an appropiate definition for indistinguishability in the phase space, have obtained a nontrivial classical counterpart of Haldane's exclusion principle.

The purpose of this Letter is first, to rederive, using a very simple interpolating argument, the occupation number distribution for geons at thermal equilibrium. Then, based on this derivation, we show that the relation for the occupation number remains invariant under a certain number of dual transformations that organize in a group and we discuss the meaning of these dualities. 


\section{Model}

A very long time ago, when the black body radiation formula was still under scrutiny, a simple argument based on interpolation has been put forward in [7]. The argument goes as follows: Let be $\bar{\varepsilon}$ the mean energy for a radiation field, averaged over time in a fixed unit volume. Using the equality of time averages and phase spase averages one can write for the mean squares values $\overline{\Delta \varepsilon^{2}}$ of the fluctuating energy that

$$
\overline{\Delta \varepsilon^{2}}=-k\left(\frac{\partial \bar{\varepsilon}}{\partial\left(\frac{1}{T}\right)}\right)_{V}
$$

Empirically, the mean square value gets contributions from low frequencies (the Rayleigh-Jeans part of the spectrum), where the mean energy $\bar{\varepsilon}=-k T$; therefore

$$
k T^{2} \frac{\partial \bar{\varepsilon}}{\partial T}=\bar{\varepsilon}^{2}
$$

It also gets contributions from high frequencies (the Wien part of the spectrum), where the mean energy $\bar{\varepsilon}=h \nu \exp \left(-\frac{h \nu}{k T}\right)$; hence

$$
k T^{2} \frac{\partial \bar{\varepsilon}}{\partial T}=h \nu \bar{\varepsilon}
$$

With the underlying assumption that the two contributions act independently and are of equal weight, relation (1) reads as

$$
k T^{2} \frac{\partial \bar{\varepsilon}}{\partial T}=\bar{\varepsilon} h \nu+\bar{\varepsilon}^{2}
$$

It is more convenient to use the dimensionless variables $n=\frac{\bar{\varepsilon}}{\varepsilon}, \frac{h \nu}{k T}=\alpha$ that lead to the differential equation:

$$
-\frac{d n}{d \alpha}=n+n^{2}
$$

The solution is

$$
n=\frac{1}{\exp \left(\alpha-\alpha_{0}\right)-1} \text { or } \bar{\varepsilon}=\frac{\varepsilon}{\exp \left(\frac{\varepsilon-\mu}{k T}\right)-1}
$$

This is, the Planck law, here with $\alpha=\frac{\mu}{k T}$, merely an integration constant. This derivation emphasises the wave-particle character of bosons.

How this reasoning can be extended for other statistics? Note that this type of interpolation works equally well for fermions. However, for fermions, as long as $\varepsilon<\mu$, the long wavelength (high temperature) is negative:

$$
\frac{d \varepsilon}{d T} \sim-\bar{\varepsilon}^{2}
$$


and accordingly, relation (5) must be modified to

$$
-\frac{d n}{d \alpha}=n-n^{2}
$$

When we take into account that $n \leq 1$, we obtain, as solution, the Fermi distribution:

$$
n=\frac{1}{\exp \left(\alpha-\alpha_{0}\right)+1}
$$

Introducing a genuine interpolation parameter $g$, i.e. a parameter ranging in the $[0,1]$ interval, one can write in a single stroke, for both fermions and bosons:

$$
-\frac{d n}{d \alpha}=n+(1-2 g) n^{2}
$$

with the solution

$$
n=\frac{1}{\exp \left(\alpha-\alpha_{0}\right)-(1-2 g)}
$$

Note that this represents also, as a particular case, for $g=\frac{1}{2}$, the Maxwell distribution function. The above interpolating formula has also been obtained in Refs.8.

At this moment we may ask what other modifications must be brought to (9) if one would allow for intermediate statistics betweeen Bose and Fermi ones on one hand, relation (9) suggests a series expension in powers of mean occupation number $n$. This is what we might expect because an interpolating statistics presumely contains more parameters and its complete characterization needs higher moments and/or higher powers of the first moment. On the other hand, higher order terms in (9) must be multiplied, for sure, by the factor $g(g-1)$, that excludes for standard fermionic $(g=1)$ and bosonic $(g=0)$ statistics any extra terms which, obviously, do not occur in (10). Limiting ourselves to the next higher order term $n^{3}$ and using again the independence and equal weights assumption (with the exception of $g(g-1)$ factor), we arrive at

$$
-\frac{d n}{d \alpha}=n+(1-2 g) n^{2}+g(g-1) n^{3}
$$

The differential equation can be solved by an elementary integration. To this purpose and for later reference we make the change of variable:

$$
w=\frac{1-n g}{n}
$$

The differential equation transforms to

$$
\frac{d w}{d \alpha}=\frac{w(w+1)}{w+g}
$$


The solution is

$$
w^{g}(w+1)^{1-g}=e^{\left(\alpha-\alpha_{0}\right)}
$$

Formulas (13) and (15) together gives implicitely the mean occupation number as function of inverse temperature. The physical meaning of the parameter $g$ can be drawn from the zero temperature limit $(\alpha \longrightarrow \infty)$ :

$$
n= \begin{cases}0 & \varepsilon>\varepsilon_{F} \\ \frac{1}{g} & \varepsilon<\varepsilon_{F}\end{cases}
$$

Therefore, $\frac{1}{g}$ represents the maximum number of particles a quatum state can accommodate. Actually, formulas (13), (15) represent exactly the particle distribution for geons at thermal equilibrium obtained by Haldane [1 from a counting argument and from equilibrium condition requirements while (16) expresses the generalized exclusion principle.

We have unfolded the derivation starting from the mean square value of the energy just to remind an historical argument 7 . Of course we could have started from the mean square value of the mean number directly. It is an well-known fact that the fluctuations in the number of bosons is super-poissonian:

$$
\overline{\Delta n^{2}}=\bar{n}+\bar{n}^{2}
$$

while for fermions is sub-poissonian:

$$
\overline{\Delta n^{2}}=\bar{n}-\bar{n}^{2}
$$

The advantage of the above derivation of fractional statistics over the standard one consists in its simplicity. The shortcoming lies in the fact the formulas for $n$ are valid only at equilibrium.

What are the transformations that leave invariant the occupation number formulas invariant, i.e. formulas (13) and (15)? To be more specific, we look for transformations for $w$ that, while preserving the substitution (13) intact, makes relation (15), or, under the same boundary condition, the differantial equation (14), form-invariant. An earlier derivation for such a tranformation [5] and a theoretical prejudice lead us to consider transformations of the form:

$$
\widetilde{w}=\frac{a w+b}{c w+d}
$$

They are known as transformations belonging to the modular group SL $(2, \mathrm{G})$, or the fractional linear group, where $\mathrm{G}$ stands for $\mathrm{C}, \mathrm{R}$ or $\mathrm{Z}$ depending on whether the coefficients $a, b, c, d$ are complex, real or integer, respectively. The group multiplication law may be represented by a $2 \times 2$ matrix $\gamma=\left(\begin{array}{ll}a & b \\ c & d\end{array}\right)$ with unit determinant $a d-c d=1$. The theoretical prejudice alluded above refers to the fact the $\mathrm{SL}(2, \mathrm{Z})$ has been found relevant for the complex conductivities in the quantum Hall effect [8]. 
We substitute Eq. (19) into the differential equation (14) and look for the coefficients $a, b, c$ and $d$ that leave the equation in same form. Rather long but straightforward algebra shows that, excepting the identity, there are only five possible tranformations of type (19) that leave the geon distribution forminvariant. These transformations $\left(t_{k}\right)$ are:

\begin{tabular}{c||c|c|c} 
& $\widetilde{w}$ & $\widetilde{g}$ & $\widetilde{\alpha}$ \\
\hline \hline$t_{1}$ & $\frac{1}{w}$ & $\frac{1}{g}$ & $-\frac{\alpha}{g}$ \\
\hline$t_{2}$ & $-\frac{i w}{i w+i}$ & $\frac{g}{g-1}$ & $\frac{\alpha}{g-1}$ \\
\hline$t_{3}$ & $-\frac{w+1}{w}$ & $\frac{g-1}{g}$ & $-\frac{\alpha}{g}$ \\
\hline$t_{4}$ & $-\frac{1}{w+1}$ & $\frac{1}{1-g}$ & $-\frac{\alpha}{1-g}$ \\
\hline$t_{5}$ & $-\frac{i w+i}{i}$ & $1-g$ & $\alpha$
\end{tabular}

The first transformation has also been remarked and derived in a different way in [5. The others are new. Note that in some transformations $\left(t_{2}\right.$ and $t_{5}$ ) the coefficients must be imaginary to comply with the requirement of unit determinant $a d-c b=1$.

These transformations deserve the name of dualities because they extend the theory from weak values of the statistical parameter $g \in[0,1]$ to strong values of the parameter $g \in[1, \infty]$ or the other way around. Indeed, starting with a weak $g \in[0,1]$, transformations $t_{1}$ and $t_{4}$ lead to formulas valid on the whole real axis for $g$ and on the range $g \in[1, \infty]$, respectively. Starting with a strong $g \in[1, \infty]$, transformation $t_{3}$ leads to formulas valid on the range $g \in[0,1]$. Transformations $t_{2}$ and $t_{5}$ map the domain of validity for $g$ (both $g$ and $\widetilde{g}$ must be necessarily positive) into themselves. The dual distributions hold true at a temperature scaled with a function of $g$, at energies below $\left(t_{2}\right.$ and $t_{5}, \alpha \geq 0$ ) or above Fermi level $\left(t_{1}, t_{2}\right.$ and $\left.t_{4}, \alpha \leq 0\right)$.

A distribution with a greater than unit g supports two interpretations:

(i) as a distribution for particles; a physical realization is the Hubbard model 9], where due to the infinite repulsion, two sites can be occupied by at most one electron. $(g=2)$ : and

(ii) as a distribution for vacancies. For instance, the generalized exclusion principle (16) states that $\frac{1}{g}$ is the maximum number of particles a quantum state can accommodate; then $\frac{1}{g}$, where $\widetilde{g}$ is the dual of $g$ under $t_{1}$ would represent the minimum number of states available for one particle.

The relationships between a specific distribution and its dual are given below

$$
\widetilde{g} \cdot n(\widetilde{\alpha}, \widetilde{g})+g \cdot n(\alpha, g)=1
$$




$$
\begin{gathered}
(\widetilde{g}-1) \cdot n(\widetilde{\alpha}, \widetilde{g})+(g-1) \cdot n(\alpha, g)=1 ; \\
(\widetilde{g}-1) \cdot n(\widetilde{\alpha}, \widetilde{g})+g \cdot n(\alpha, g)=1 \\
\widetilde{g} \cdot n(\widetilde{\alpha}, \widetilde{g})+(g-1) \cdot n(\alpha, g)=1 ; \\
n(\widetilde{\alpha}, \widetilde{g})+n(\alpha, g)=0
\end{gathered}
$$

The last relationship is somehow peculiar. Apparently, due to $g \longrightarrow 1-g$ transformation, it seems to convert the distribution for fermions or near fermions $\left(g \geq \frac{1}{2}\right)$ into the distribution for bosons or near bosons $\left(g \leq \frac{1}{2}\right)$ and vice-versa, but the dual $n(\widetilde{\alpha}, \widetilde{g})$ of $n(\alpha, g)$ is, in this case, negative

The above dualities impose constraints on the expansion coefficients of thermodynamic functions. For instance, the duality (20a) leads to a vanishing zero temperature heat capacity (Refs. 5-8). A detailed derivationand analysis of these constraints are relegated to an extended paper. Here we are content to remark that all five transformations are needed for these transformations to close upon themselves, i.e. to form a group. We think the group property is the most remarkable feature and we give below the table for the group composition law:

\begin{tabular}{c||c|c|c|c|c|c} 
& $t_{0}$ & $t_{1}$ & $t_{2}$ & $t_{3}$ & $t_{4}$ & $t_{5}$ \\
\hline \hline$t_{0}$ & $t_{0}$ & $t_{1}$ & $t_{2}$ & $t_{3}$ & $t_{4}$ & $t_{5}$ \\
\hline$t_{1}$ & $t_{1}$ & $t_{0}$ & $t_{3}$ & $t_{2}$ & $t_{5}$ & $t_{4}$ \\
\hline$t_{2}$ & $t_{2}$ & $t_{4}$ & $t_{0}$ & $t_{5}$ & $t_{1}$ & $t_{3}$ \\
\hline$t_{3}$ & $t_{3}$ & $t_{5}$ & $t_{1}$ & $t_{4}$ & $t_{0}$ & $t_{2}$ \\
\hline$t_{4}$ & $t_{4}$ & $t_{2}$ & $t_{5}$ & $t_{0}$ & $t_{3}$ & $t_{1}$ \\
\hline$t_{5}$ & $t_{5}$ & $t_{3}$ & $t_{4}$ & $t_{1}$ & $t_{2}$ & $t_{0}$
\end{tabular}

where $t_{0}$ denotes the identity. The left multiplication corresponds to entries along column, the right multiplication to entries along rows. It is worthy to note that transformations $t_{j}, j=3,4$ are inverse of each other, a fact that can also be seen from rels. (20.3), (20.4)

\section{Conclusions}

To sum up, we have proved that the fractional statistics distribution is invariant to a finite, nonabelian subgroup of the fractional linear group.

We have discussed the duality in terms of transformations that, while leaving the theory invariant, relate two different "coupling constant" $g$ regimes. There exists, also, another meaning of duality in this letter, as illustrated, for instance, in relation (4), namely, the wave-particle duality. The first term in the RHS of rel. (4) features the particle-like character of bosons and the second, the 
wave-like character. The fact the two terms simply add togather expresses the complementarity of the two features. In standard quantum mechanics, this duality is embodied in the usual momentum-position commutator. We may wonder if the newly introduced term $g(g-1) n^{3}$ for fractional statistics, in relation (12) signals the existence, in an analogous way, of another level of duality.

In view of the above remarks it is tempting to put forward the following conjecture: there must exist another type of duality, in the sense above, but between vortices and particles (as in some models for anyons [11]). The conjecture is rather speculative at the moment. We hope to return with a more rigorous statement in the next future.

\section{Acknoledgement}

The autor thanks Gr. Ghika for critical reading of the paper.

\section{References}

[1] F.D.M. Haldane - Phys. Rev. Lett. 67, 937 (1991)

[2] Y. S. Wu - Phys. Rev. Lett. 73, 922 (1994)

[3] F. Buyukkihc and D. Demirhan - Eur. Phys. Journ. B!4, 704 (2000)

[4] F. Buyukkihc, H. Uncu and D. Demirhan - cond-mat/0103560

[5] C. Nayak and F. Wilczek - Phys.Rev. Lett. 73, 2740 (1994)

[6] T. H. Hansson. S. B. Isakov. J. M. Leinaas and U. Lindstrom - quant-ph/0003121 quant-ph/ 0004108

[7] A. Einstein - Ann. Phys. 19, 132, 549 (1905)

[8] J. Acharya and M. Narayanaswamy, J. Phys. A: Math. Gen. 27, 7247 (1994)

A. Polychronakos, Phys. Lett. B365, 202 (1996)

[9] C. A. Lutken and G. G. Ross - Phys. Rev. B45 11837 (1992); B. P. Dolan -condmat/0002228

[10] H. Tasaki - cond-mat/9512169

[11] For a review on anyons see, for instance, S. Rao-hep-th/9209066; for vortexparticle duality see A.A. Zee-cond-mat/9501022 